Monatsschr Kinderheilkd 2009 · 157:422-422

DOI 10.1007/s00112-009-1997-7

Online publiziert9. April 2009

(c) Springer Medizin Verlag 2009

\author{
J. Martinius ${ }^{1} \cdot$ D. Reinhardt ${ }^{2}$ \\ ${ }^{1}$ Klinik für Kinder- und Jugendpsychiatrie, Psychosomatik und Psychotherapie, \\ Ludwig-Maximilians-Universität München \\ ${ }^{2}$ Dr. von Haunersches Kinderspital, Ludwig-Maximilians-Universität München
}

\title{
Kind und Medien
}

Das Thema „Kind und Medien“ bezeichnet ein weites Feld, auf dem sich mancherlei praktische und wissenschaftliche Interessen zusammenfinden: die Medienwelt und ihre Wissenschaft selbst, Politik, Pädagogik, Psychologie und eben auch die Medizin. Denn die Wirkungen von Medien haben gesundheitliche Relevanz bis hin zur Verursachung von Schäden (u. a. Haltungsschäden, Übergewicht, Sucht). Für Kinder- und Jugendärzte ergeben sich daraus kurative und präventive Aufgaben. Voraussetzung für ihre Erfüllung sind spezielle Kenntnisse.

\section{จ Ärzte müssen krank machende Wirkungen der Medien kennen und ihnen entgegentreten}

Medien erreichen Kinder und Jugendliche mehr oder weniger, ob wir das als Ärzte oder Eltern wollen oder nicht. Und es müssen auch nicht nur die negativen Folgen des Konsums sein, an denen wir unser Denken und Handeln fest machen. Gleichwohl sind wir als Ärzte aufgefordert, krank machende Wirkungen zu kennen und ihnen zum Wohle unserer Patienten entgegenzutreten. Um Kompetenz in diesem Bereich zu aktualisieren und weiterzuentwickeln, entstand dieses Heft mit ausgewählten Aufsätzen zum Thema. Bestandsaufnahmen und Wirkmechanismen sollten gleichermaßen Darstellung finden.

Gibt es einen „richtigen“ Gebrauch und wie kann das natürliche Interesse von Kindern dorthin gelenkt werden? Wie steht es heute um das nach wie vor von Kindern meistgenutzte Medium Fernsehen? Es ist schon längere Zeit bekannt, dass ausgerechnet die verwundbaren, die psychisch und sozial belasteten Kinder und Jugendlichen diejenigen sind, welche die meisten Sendungen mit belastendem Inhalt konsumieren. Sexuelle Gewalt, gerade auch gegen Kinder, ist eines der traurigsten Phänomene des Mediengeschehens. Und pathologischer Mediengebrauch als Tor zu Abhängigkeit und Sucht muss uns ebenso tief bekümmern. Kinder werden von Medien aber auch in voyeuristischer Weise benutzt, um Gewinne zu steigern. Kinderschutz hat hier eine wichtige Aufgabe.

Als Herausgeber dieses Schwerpunktheftes hoffen wir, eine informative und aktuell nutzbare Auswahl von Artikeln getroffen zu haben und danken den Autorinnen und Autoren für ihre Beteiligung an diesem Vorhaben.

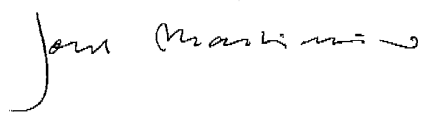

J. Martinius

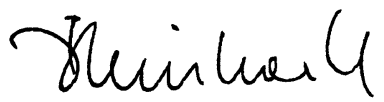

D. Reinhardt

\section{Korrespondenzadresse}

Prof. Dr. J. Martinius

Klinik für Kinder- und Jugendpsychiatrie, Psychosomatik und Psychotherapie, Ludwig-Maximilians-Universität München Waltherstraße 23, 80337 München martinius@Irz.uni-muenchen.de 